\title{
VALIDATION OF WIMS11 FOR SMALL MODULAR REACTOR ANALYSIS
}

\author{
Peter Smith $^{1}$, Glynn Hosking ${ }^{1}$, Brendan Tollit ${ }^{1}$, Ben Lindley ${ }^{1}$, Andrew Cox ${ }^{1}$, Ray Perry ${ }^{1}$, \\ Tim Ware ${ }^{1}$, Robert Mason ${ }^{1}$ and Magda Stefanowska ${ }^{1}$
}

\author{
${ }^{1}$ Wood Nuclear \\ Queen Mother Square, Poundbury, Dorset DT1 3BW, UK \\ Peter.Smith2@woodplc.com,
}

\begin{abstract}
The WIMS (Winfrith Improved Multigroup Scheme) reactor physics code is actively being developed for whole core modelling of a range of Small Modular Reactor types including the Pressurized Water Reactor (PWR), High Temperature Reactor (HTR), and Liquid Metal Cooled Fast reactor (LMFR). These developments include the capability for whole core multiphysics modelling with neutronics and thermal hydraulic feedback, as well as methods to determine the power deposition from neutron and gamma heating. Flux solutions are obtained using a wide variety of deterministic methods including diffusion theory, SP3, and full transport with the method of characteristics and Sn discrete ordinates methods, as well as multi-group Monte Carlo methods. The SP3 method allows both steady state and time dependent transient solutions by solving the time dependent SP3 equations. A wide variety of nuclear data libraries are available with WIMS including data from the JEF3.3, ENDF/B-VII.0 and CENDL3.1 nuclear data evaluations.

This paper presents validation of the latest version of the WIMS code, WIMS11, for PWR and HTR systems. Comparisons are made against physics data obtained from the OECD/NEA PWR Watts Bar multi-physics benchmark and the IAEA HTR-10 benchmark, as well as neutron and gamma heating experiments that took place on the NESSUS reactor at Winfrith in the United Kingdom. In each case, validation of WIMS has been obtained by comparison either against measured data, or results provided by other benchmark participants that have been obtained with alternative deterministic or Monte Carlo methods.
\end{abstract}

KEYWORDS: WIMS, VALIDATION, PWR, HTR, HEATING

\section{INTRODUCTION}

For Small Modular Reactors (SMRs), improved high fidelity predictive modelling is desirable to facilitate reactor licensing and operation, being particularly important for innovative reactor design features (e.g. soluble boron free SMR) and accident tolerant fuels for which experimental or prototype facilities may be more limited. The high fidelity codes for the design and analysis of SMR cores requires extensive verification and validation, using the available real plant experimental data to ensure that the capabilities of the codes systems are fully tested in the analysis of the core-coupled neutronics and thermal hydraulic interaction. Addressing the validation needs of such high fidelity multi-physics calculation schemes is therefore of importance, and is being addressed through international projects such as the Tennessee Valley Authority ( TVA) Watts Bar Unit 1 Multi-Physics Multi-Cycle Benchmark [1]. 
Developed by the ANSWERS Software Service in Wood, the WIMS reactor physics code has been under development since the late 1960s [2]. The latest version of the WIMS code, WIMS11 [3], has been developed, benchmarked and validated for reactor physics analysis of PWRs [3], HTRs and LMFRs [4]. This paper presents validation of this capability for multi-physics analysis of SMRs. A number of elements are presented, which in combination, ultimately provide this capability:

- Development of a whole core analysis methodology. This approach is based on a capability to define a whole core model in terms of materials, geometry and mesh structures for resonance shielding, burnup, flux and thermal calculations. Control rods can be defined, and their withdrawal modified, on a single rod, cluster, bank or group basis. Few group resonance shielded cross sections are generated using either equivalence theory, or the more advanced sub-group theory method, with corrections applied to produce homogenized assembly data using the super homogenization approach.

- The few group homogenized cross section data are used in a variety of whole core flux solvers, depending on the desired accuracy and performance. Reference transport theory calculations can be performed using either the method of characteristics or the discrete ordinates Sn method, while more approximate but faster running solution can be obtained using diffusion theory or an SP3 solution.

- Development of a coupled neutronic-thermal hydraulic calculation scheme. This methodology has been achieved by the development of a sub-channel thermal hydraulic solver which is fully incorporated within the WIMS code [5].

- Development of a coupled neutron and gamma heating calculation scheme. This methodology has been achieved by extending the nuclear data capability within the WIMS code to produce photon cross section data for production, scattering and absorption processes. The photon cross section data is used to generate an inhomogeneous source, which can be applied with many of the flux solvers within WIMS to calculate photon transport.

\section{TVA WATTS BAR MULTI-PHYSICS BENCHMARK}

\subsection{Benchmark Description}

This study describes validation of WIMS on TVA Watts Bar Unit Cycle 1 (WBN1), with specific focus on start-up Zero Power Physics Tests (Exercise 1). The specifications for this benchmark activity were selected from a series of benchmark problems [6] developed by the Consortium for the Advanced Simulation of Light Water Reactors (CASL), a United States Department of Energy Innovation Hub focused on developing and applying advanced modeling and simulation capabilities to improve the operations and safety of light water reactors. Detailed description of fuel rod, fuel assemblies, control rod, Pyrex (borosilicate glass), guide tubes, spacer grid, thimble plug, and instrument thimble are provided. Details of the core loading patterns, radial core and numerous vessel components are also outlined. The measured plant data for Exercise 1 is also provided [1].

Exercise 1 is based on problem 6 of the Virtual Environment for Reactor Applications (VERA) benchmark specification (WBN Cycle 1 BOC HFP). The purpose of this exercise is to predict the start-up Zero Power Physics Tests (ZPPT) results for Cycle 1 of a Westinghouse 4-loop physical reactor with a 17 x 17 fuel assembly design. The tests include measurement of critical boron concentration, isothermal temperature coefficient, control rod bank worth's, and soluble boron worth. The tests are performed at zero power no xenon conditions without thermal hydraulic or fuel temperature feedback. Successful completion demonstrates the capability to predict the eigenvalue and core reactivity coefficients without thermal hydraulic feedback or depletion. 


\subsection{Methodology and Results}

The WIMS neutronic calculation methodology for whole core SMR's employs a new module, GEOM, to define a whole core model in terms of materials and geometry, and calculation method options and mesh structures for the different types of calculation (resonance shielding, thermal, burnup and flux). Few group resonance shielded cross sections are generated for each material set using either equivalence theory, or the more advanced sub-group theory method, with corrections applied to produce homogenized assembly data using the super homogenization approach. Few group homogenized cross section data are used with a variety of whole core flux solvers. Reference transport theory calculations can be performed using the method of characteristics, the CACTUSOT module with a specific once through tracking algorithm, while a more approximate but faster running solution can be obtained with the MERLIN module using diffusion theory or an SP3 solution.. A quarter core model of the TVA Watts Bar multi-physics benchmark has been established using the WIMS whole core modelling capability, as shown in Figure 1 below.

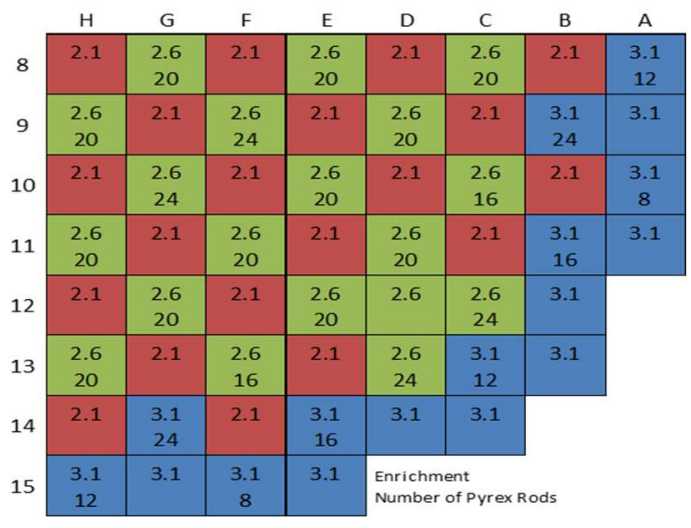

(A)

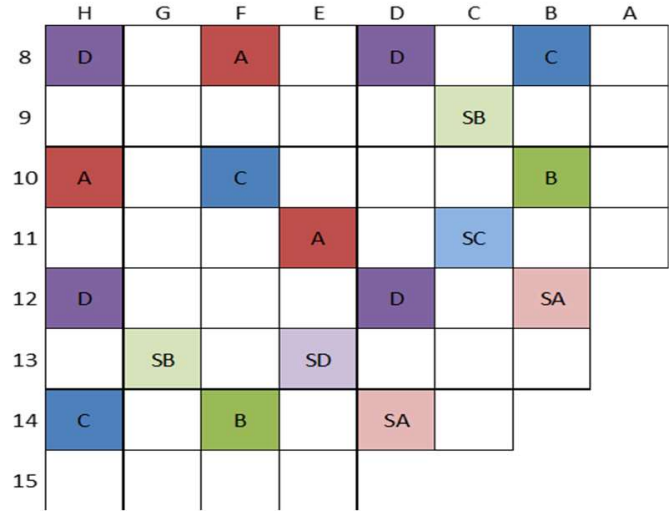

$(\mathrm{R})$

Figure 1. Assembly, Poison (A) and Control Rod Layout (B) - 1/4 Symmetry.

The WIMS multi-physics calculation methods for LWR SMR's employs a slowing down calculation using sub-group theory to generate 172-group cross sections, based on the JEFF-3.1.2 nuclear data library. The PERSEUS module is used to calculate collision probabilities with a multi-cell collision probability method to homogenize and condense to 22 energy groups, and an SP3 calculation in XYZ geometry using the MERLIN module within WIMS is used for the core flux solution. Suitable homogenization corrections used to compensate for transport and mesh effects were determined through the application of the super homogenization method with the CACTUS method of characteristics method used to prepare a reference transport theory solution. The calculated results for Exercise 1 of the TVA Watts Bar multi-physics benchmark are shown in Table I below.

Table I. Zero Power Physics Test Results- Exercise 1

\begin{tabular}{|c|c|c|c|c|}
\hline Parameter & Measured & $\begin{array}{c}\text { WIMS } \\
\text { Prediction }\end{array}$ & Difference & Units \\
\hline Critical Boron at HZP (ppm) & 1293 & 1282 & -11 & $\mathrm{ppm}$ \\
\hline Bank D Worth (pcm) & 1342 & 1385 & +43 & $\mathrm{pcm}$ \\
\hline Bank C Worth (pcm) & 940 & 975 & +35 & $\mathrm{pcm}$ \\
\hline Bank B Worth (pcm) & 871 & 883 & +12 & $\mathrm{pcm}$ \\
\hline
\end{tabular}




\begin{tabular}{|c|c|c|c|c|}
\hline Bank A Worth $(\mathrm{pcm})$ & 830 & 875 & +45 & $\mathrm{pcm}$ \\
\hline Bank SD Worth $(\mathrm{pcm})$ & 495 & 501 & +6 & $\mathrm{pcm}$ \\
\hline Bank SC Worth $(\mathrm{pcm})$ & 494 & 500 & +6 & $\mathrm{pcm}$ \\
\hline Bank SB Worth $(\mathrm{pcm})$ & 1048 & 1066 & +18 & $\mathrm{pcm}$ \\
\hline Bank SA Worth $(\mathrm{pcm})$ & 431 & 444 & +13 & $\mathrm{pcm}$ \\
\hline Total Rod Worth $(\mathrm{pcm})$ & 6451 & 6667 & +216 & $\mathrm{pcm}$ \\
\hline Boron Worth $(\mathrm{mN} / \mathrm{ppm})$ & -10.8 & -10.0 & -0.7 & $\mathrm{mN} / \mathrm{ppm}$ \\
\hline
\end{tabular}

The control rod worths, critical boron concentration and boron worth are well predicted by WIMS, although there is a trend to overestimate the value of the control rod worth.

\section{HTR-10 BENCHMARK}

\subsection{Benchmark Description}

The HTR-10 reactor is China's first major development in modular gas cooled HTR technology. This validation test is taken from the revised version of the IAEA benchmark "Evaluation of High Temperature Gas Cooled Reactor Performance" [7], which was developed under the IAEA research co-ordinated programme (RCP) 5. Experimental results are available from the commissioning experiments for the core height at which first-criticality was achieved $(123.06 \mathrm{~cm})$. In this test, the critical height calculated in a two dimensional RӨZ WIMS pebble bed modular reactor model is compared with the measured critical height. It can be noted that there is some uncertainty in the measured critical height because the critical height was deduced from the number of pebbles loaded into the core and the assumed packing density, which may be different from the actual packing density. For the initial reactor loading, upon which this calculation is based, the core was loaded with a mix of 57\% fuel pebbles and $43 \%$ graphite dummy pebbles. Each of the pebbles has a diameter of $6 \mathrm{~cm}$. The fuel is uranium dioxide and the fuel uranium enrichment is $17 \%$ by mass. Each fuel pebble contains 5 grams of metal fuel, which is contained within approximately 8335 coated particles. Within the commissioning core, the mixture of fuel and carbon pebbles occupies a space with diameter 1.8 meters and a height of approximately 1.8 meters. This pebble filled region is surrounded by radial and axial reflectors. Detailed geometry descriptions and plans of the core can be found in [7], and a plan view of the HTR-10 reactor core is shown below in Figure 2.

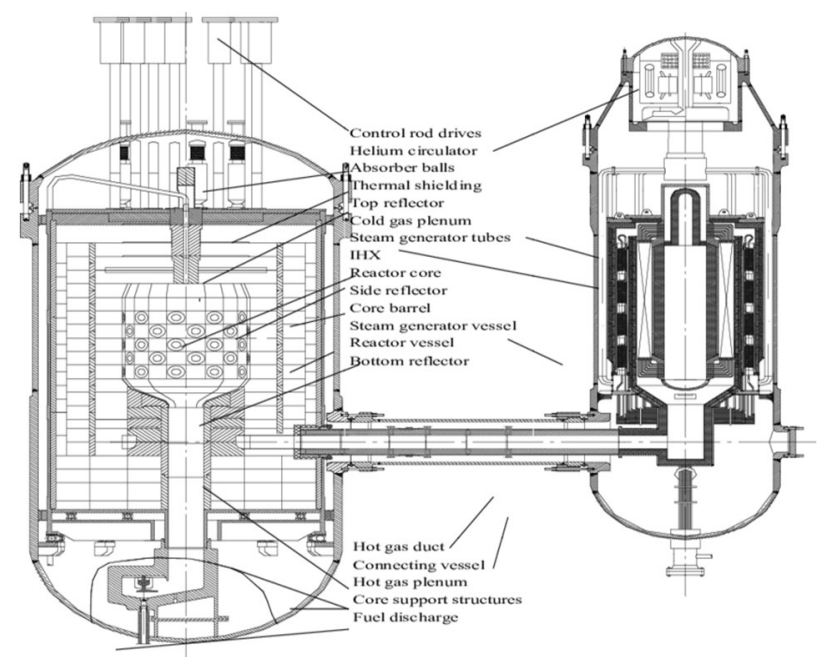

Figure 2. Plan View of the HTR-10 Reactor. 


\subsection{Methodology and Results}

The WIMS neutronics calculation methodology for particle fueled High Temperature Reactors employs a slowing down calculation using sub-group theory to generate 172-group cross sections, based on the JEFF3.1.2 nuclear data library. The PROCOL module is used to calculate collision probabilities for the spherical fuel particle and fuel pebble geometry, and a multi-cell collision probability method is used for the homogenization of the fuel and dummy graphite pebbles in the whole core model. The SP3 method in RZ geometry is used to condense to 22 groups, and an SP3 calculation in ROZ using the MERLIN module within WIMS is used for the core flux solution. Suitable corrections used to compensate for transport, condensation and mesh effects were determined through a series of scoping studies. The calculated results are shown in Table II below. The loaded core height is defined as the height to which the core is filled with pebbles. The uncorrected values of k-effective are those obtained with an unrefined radial and axial mesh, and in a condensed 22 energy structure. The corrections are formed as the sum of terms that account for energy group condensation effects, mesh effects and transport theory effects (since the core calculations use a SP3 theory solution). The corrections also include factors that account for modelling the cone in discrete steps instead of as a continuous structure and not modelling reflector penetrations explicitly. These last corrections are derived from calculations performed with the MONK Monte Carlo code [8].

Table II. WIMS Results for the HTR-10 Benchmark

\begin{tabular}{|c|c|c|c|}
\hline $\begin{array}{c}\text { Loaded Core Height } \\
(\mathrm{cm})\end{array}$ & $\begin{array}{c}\text { k-effective } \\
\text { uncorrected }\end{array}$ & $\begin{array}{c}\text { Correction } \\
(\mathrm{pcm})\end{array}$ & $\begin{array}{c}\text { k-effective } \\
\text { corrected }\end{array}$ \\
\hline 130.09 & 1.04800 & -1837 & 1.03011 \\
\hline 120.08 & 1.01884 & -1870 & 0.99983 \\
\hline 110.07 & 0.98475 & -1841 & 0.96576 \\
\hline
\end{tabular}

The critical height prediction from the above table, for a value of k-effective $=1.0$, is $120.03 \mathrm{~cm}$. The measured critical height was $123.06 \mathrm{~cm}$. Therefore, the difference between calculation and measurement is $3.03 \mathrm{~cm}$. A curve of MONK k-effective predictions was plotted, and the gradient of this curve at the critical height was used to deduce a worth differential of $214 \mathrm{pcm} / \mathrm{cm}$. Therefore, the $3.03 \mathrm{~cm}$ difference between calculation and measurement corresponds to an over-prediction in the absolute core k-effective of $648 \mathrm{pcm}$ against measurement. A limited set of HTR-10 benchmark calculations has been performed with the MONK Monte Carlo code in order to obtain an estimate of the critical height. These calculations used the BINGO data library, which includes bound-carbon scattering data. The results from these calculations are shown in Table III below.

Table III. MONK Results for the HTR-10 Benchmark

\begin{tabular}{|c|c|}
\hline Case & $\begin{array}{c}\text { MONK } \\
\text { k-effective }\end{array}$ \\
\hline $\begin{array}{c}\text { Full height core, full cone modelled, } \\
\text { reflector penetrations explicitly represented }\end{array}$ & $1.1294 \pm 0.0010$ \\
\hline As above but fuelled core height $=150 \mathrm{~cm}$ & $1.0681 \pm 0.0010$ \\
\hline As above but fuelled core height $=125 \mathrm{~cm}$ & $1.0128 \pm 0.0010$ \\
\hline As above but fuelled core height $=100 \mathrm{~cm}$ & $0.9057 \pm 0.0010$ \\
\hline
\end{tabular}

A fit to the MONK results predicts a critical core fuel height $(\mathrm{k}$-effective $=1.0)$ of $120.9 \mathrm{~cm} \pm 0.8 \mathrm{~cm}$. The measured critical height was $123.06 \mathrm{~cm}$, a difference of $2.16 \mathrm{~cm}$. Using a worth differential of $214 \mathrm{pcm} / \mathrm{mm}$, this represents an over prediction in absolute core k-effective against measurement of $450 \pm 171 \mathrm{pcm}$. The 
WIMS result is therefore in good agreement with that from MONK. There is reasonable agreement between the measured and calculated critical height, considering that the transport and condensation corrections are taken from a one dimensional geometry model and the transport correction has been taken from a 22 group rather than a fine group calculation. The corrections for mesh, condensation and transport effects have been confirmed as reasonable by making comparisons with a 172 broad group MONK calculation. The WIMS results have also been confirmed to be in reasonable agreement with those from equivalent continuous energy MONK calculations.

\section{NEUTRON AND GAMMA HEATING BENCHMARK}

\subsection{Benchmark Description}

Energy deposition by radiation is an important consideration in the design and operation of both nuclear reactors and fuel transport flasks. For example, in a gas cooled graphite moderated reactor the radiolytic oxidation of the graphite, which is dependent on the total radiation energy deposition, causes corrosion of the graphite components and reduces their integrity. For fuel transport flasks, knowledge of the energy deposited in the flask walls and its contents is important for heat transfer analysis. Validation of the WIMS methods has been performed against a benchmark measurement of heat deposition made in the NESSUS facility of the NESTOR reactor using a graphite micro-calorimeter. NESTOR was a water cooled, graphite moderated reactor with a maximum operating power of $30 \mathrm{~kW}$. It consisted of an annular ring of some 26 fuel elements, each comprising 16 plates of $20 \% \mathrm{U} / \mathrm{Al}$ alloy plates at $80 \% \mathrm{U} 235 / \mathrm{U}$ ratio. These are cooled by light water which flows over the fuel plates and provides some neutron moderation. The fuel is surrounded by a graphite reflector and there is a $602 \mathrm{~mm}$ diameter central graphite thermal column. The reactor is controlled by the movement of calibrated neutron absorbing control plates located just outside the annulus of fuel. A plan view of the NESTOR core is shown in Figure 3.

The NESSUS facility is a vertical tube passing through the middle of the central thermal column. An aluminium thimble is inserted into this tube, through which samples up to $43 \mathrm{~mm}$ diameter can be introduced into the nuclear center of the reactor. Measurements of nuclear heating rates were made using four ULRC Mk II calorimeters manufactured to the same design but constructed from different materials. Three of the calorimeters used the same material for sample and baffles; the three materials were graphite, aluminium and iron. The fourth comprised a graphite sample with iron baffles and was constructed to study the effect of electron migration. This analysis relates to the graphite calorimetry measurements only. The total heating was measured directly using a range of detectors. The gamma heating was also measured directly but the neutron heating was inferred using measured and calculated fast reactor reaction rates.

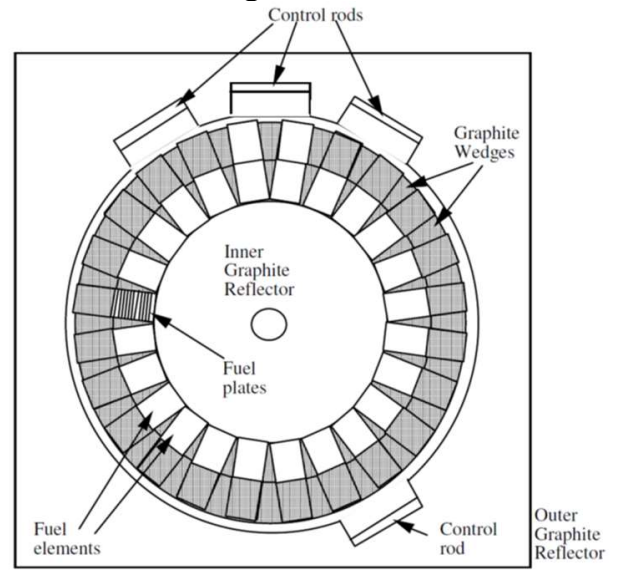

Figure 2. Plan View of the NESSUS Facility in the NESTOR Reactor. 


\subsection{Methodology and Results}

Two calculation models in RZ geometry were established in WIMS, Model A where no detector region was included, and Model B where a detector region was included. Inclusion of the gamma data was achieved using the PHODAT module in WIMS to generate microscopic and macroscopic photon interaction cross sections and photon source cross sections for use in the calculation of gamma heat distributions. The SOURCE module was used to create a fixed gamma source representation. A neutron transport calculation and flux solution was obtained using either the method of characteristics (the CACTUS module) or the SP3 approximation (the MERLIN module). The gamma flux solution was obtained using either the CACTUS, SP3, or GAM module which utilizes a Monte Carlo method. It can be noted that both the MERLIN and CACTUS modules have no suitable anisotropic scatter representation for the gamma transport. Initial results, obtained for the graphite detector with data from the JEFF 3.1.2 nuclear data evaluation, are shown in Table IV below.

Table IV. Heating (micro W/g) : Graphite Detector

\begin{tabular}{|c|c|c|c|}
\hline Method & Total & Gamma & Neutron \\
\hline Experiment & $\begin{array}{c}25.42 \\
(\mathrm{sd} 2 \%)\end{array}$ & $\begin{array}{c}21.55 \\
(\operatorname{sd} 3.9 \%)\end{array}$ & $\begin{array}{c}4.07 \\
(\operatorname{sd} 3.9 \%)\end{array}$ \\
\hline \multicolumn{4}{|c|}{ Calculation - Model A (Neutron-Gamma) } \\
\hline CACTUS-CACTUS & 21.32 & 17.48 & 3.84 \\
\hline SP3-SP3 & 21.50 & 17.57 & 3.93 \\
\hline SP3-GAM & 26.02 & 22.09 & 3.93 \\
\hline \multicolumn{4}{|c|}{ Calculation - Model B (Neutron-Gamma) } \\
\hline CACTUS-CACTUS & 21.04 & 17.12 & 3.92 \\
\hline SP3-GAM & 28.16 & 24.34 & 3.82 \\
\hline
\end{tabular}

The WIMS SP3 and CACTUS predictions are in good agreement for both neutron and gamma heating. The WIMS SP3 result is in good agreement with experiment for neutron heating, and the WIMS GAM calculation gives good accuracy for gamma heating. As expected, there is an underprediction for gamma heatung when the detector geometry is not represented, while the prediction of gamma heating is increased when the detector is modelled. Generally, the gamma heating is over predicted due to the WIMS nuclear data library containing saturated gamma source data. Several means were investigated to improce Model B including allowing both radial and axial variation in the gamma source in the fuel ring, and also including $\mathrm{U}^{238}$ within the fuel ring. Updated results for Model B are given in Table $\mathrm{V}$ below. As a result of the modelling improvements, the WIMS prediction of gamma heating has been reduced by $\sim 2$ micro $\mathrm{W} / \mathrm{g}$. and now shows very good agreement with the measured value.

Table V. Heating for the Improved Model B (micro W/g) : Graphite Detector

\begin{tabular}{|c|c|c|c|}
\hline Method & Total & Gamma & Neutron \\
\hline Experiment & $\begin{array}{c}25.42 \\
(\mathrm{sd} 2 \%)\end{array}$ & $\begin{array}{c}21.55 \\
(\mathrm{sd} 3.9 \%)\end{array}$ & $\begin{array}{c}4.07 \\
(\mathrm{sd} 3.9 \%)\end{array}$ \\
\hline \multicolumn{4}{|c|}{ Calculation - Model B (Neutron-Gamma) } \\
\hline CACTUS-CACTUS & 26.01 & 22.14 & 3.87 \\
\hline SP3-GAM & 24.36 & 20.85 & 3.51 \\
\hline
\end{tabular}




\section{CONCLUSIONS}

The latest version of the WIMS reactor physics code, WIMS11, is actively being developed for whole core modelling of a range of Small Modular Reactor types including the Pressurized Water Reactor (PWR), High Temperature Reactor (HTR), and Liquid Metal Cooled Fast reactor (LMFR). .Validation of WIMS11 includes comparisons made against physics data obtained from the OECD/NEA PWR Watts Bar multiphysics benchmark and the IAEA HTR-10 benchmark, as well as neutron and gamma heating experiments that took place on the NESSUS reactor at Winfrith in the United Kingdom. Results are presented that are in good agreement with benchmark measurements for control rod worths, critical boron concentration and boron worth (LWR), critical height (HTR), and neutron and gamma heating.

\section{ACKNOWLEDGMENTS}

This work was part funded by the UK government's Department for Business, Energy and Industrial Strategy, as part of the Nuclear Innovation Programme (Tender Reference Number (TRN) 1404/12/2017).

\section{REFERENCES}

1. A. Godfrey, S. Palmtag, F. Franceshini, L. Moloko, T. Saad, M. Avramova, and K. Ivanov, "TVA Watts Bar Unit 1 Multi-Physics Multi-Cycle Benchmark," Draft Benchmark Specifications (April 2019).

2. J. R. Askew, F. J. Fayers and P. B. Kemshell, "A General Description of the Lattice Code WIMS", BNES Vol. 5 No. 4, P564, 1966.

3. B. A. Lindley, T. D. Newton, J. G. Hosking, P. N. Smith, D. J. Powney, B. Tollit, and P. J. Smith. "Release of WIMS 10: A Versatile Reactor Physics Code for Thermal and Fast Systems." In Proceedings of ICAPP 2015, Nice, France (May 2015).

4. U. Davies, B. Lindley, B. Tollit, and E. Shwageraus. "Whole-Core Validation of the Superphenix Reactor using WIMS11 and an Investigation into a Hybrid RZ-HEX SP3 Calculation Route." In Proceedings of M\&C2019, Portland, Oregon, USA (2019).

5. B. Tollit, "Development of a Subchannel Model within the ANSWERS Software Service WIMS Reactor Physics Code," in Proceedings of PHYSOR 2018, Cancun, Mexico, (April 2018).

6. A. Godfrey, "VERA Core Physics Benchmark Progression: Problem Specifications," CASL report, CASL-U-2012-0131-004, Revision 4, August 2014.

7. "Evaluation of High Temperature Gas Cooled Reactor Performance: Benchmark Analysis Related to Initial Testing of the HTTR and HTR-10", IAEA-TECDOC-1382, November 2003.

8. S. Richards, G. Dobson, T. Fry, D. Hanlon, D. Long, R. Perry, P. Smith, F. Tantillo, and T. Ware, "Recent Developments to the MONK Monte Carlo Code for Criticality Safety and Reactor Physics Analysis," In Proceedings of ICNC 2019, Paris, France (September 2019). 